\title{
Effect of a Gluten-Free Diet on Albuminuria in Children with Newly Diagnosed Celiac Disease
}

\author{
Jeremiah Levine ${ }^{a}$ Leora Hauptman ${ }^{a}$ Libia Moy ${ }^{a}$ Howard Trachtman ${ }^{b}$ \\ aDivision of Gastroenterology, Department of Pediatrics, NYU Langone Health, New York, NY, USA; ${ }^{\text {D Divisions }}$ \\ Nephrology, Department of Pediatrics, NYU Langone Health, New York, NY, USA
}

\section{What is Known on This Subject}

- Celiac disease $(\mathrm{CD})$ is mediated by enterocyte release of zonulin. The receptor for zonulin is expressed on podocytes, raising the possibility that CD patients may have increased glomerular permeability to albumin.

\section{What This Study Adds}

- Children with newly diagnosed celiac disease have low-grade albuminuria that is numerically higher than in controls. Implementation of a gluten-free diet was associated with a reduction in albuminuria that was more pronounced in the subgroup with a baseline albumin $\mathrm{Cr} \geq 10 \mu \mathrm{g} / \mathrm{mg}$.

\section{Keywords}

Celiac disease · Glomerular permeability · Gluten-free diet · Albuminuria

\section{Abstract \\ Background and Objectives: Altered gastrointestinal per- meability in celiac disease (CD) is mediated by zonulin. The receptor for zonulin is expressed on podocytes. Therefore, we tested the effect of a gluten-free diet (GFD) on albumin- uria in pediatric patients with newly diagnosed CD. Meth- ods: We performed a cohort study comparing urinary albu- min $(\mu \mathrm{g}): \mathrm{Cr}(\mathrm{mg})$ ratio (ACR) in CD patients versus controls}

and in response to a GFD. Results: Children with $C D(n=46)$ had higher ACR than controls $(n=21), 20.2 \pm 5.6$ versus 8.4 $\pm 1.1 \mu \mathrm{g} / \mathrm{mg}, p=0.16$ and exceeded $30 \mu \mathrm{g} / \mathrm{mg}$ (microalbuminuria cutoff) in $7 / 46$ cases. Seventeen patients had a follow-up assessment (interval $6.1 \pm 0.7$ months) on a GFD. Baseline ACR was $20.7 \pm 5.2$ that fell to $10.4 \pm 1.5 \mu \mathrm{g} / \mathrm{mg}, p=$ 0.035 . Conclusion: Children and adolescents with newly diagnosed CD have low-grade albuminuria that is numerically higher than controls and that declined after implementation of a GFD. CD may be associated with reversible defects in the glomerular barrier.

(c) 2021 The Author(s)

Published by S. Karger AG, Basel

$\begin{array}{ll}\text { karger@karger.com } & \begin{array}{l}\text { (c) } 2021 \text { The Author(s). } \\ \text { Published by S. Karger AG, Basel }\end{array} \\ \text { www.karger.com/gdz } & \begin{array}{l}\text { This is an Open Access article licensed under the Creative Commons } \\ \text { Attribution-NonCommercial-4.0 International License (CC BY-NC) } \\ \text { (http://www.karger.com/Services/OpenAccessLicense), applicable to } \\ \text { the online version of the article only. Usage and distribution for com- } \\ \text { mercial purposes requires written permission. }\end{array}\end{array}$




\section{Background}

Celiac disease (CD) is a chronic immune-mediated condition that develops in genetically predisposed individuals $[1,2]$. CD is characterized by an enteropathy and in a subset of patients, other extra-gastrointestinal manifestations in association with circulating autoantibodies [3-5]. An important element in the pathophysiology of $\mathrm{CD}$ is a change in gut permeability caused by a loss of integrity of the paracellular tight junctions $[6,7]$. It is speculated that once the integrity of the intestinal barrier is breached and permeability increases, transit of the toxic gliadin peptides toward the lamina propria is facilitated, amplifying the immunological chain reaction [6-8]. A key participant in this process is zonulin, an intestinal peptide that regulates the tight junctions. Circulating levels of zonulin are elevated in CD and reduced upon instituting a gluten-free diet (GFD) [9]. Mechanistically, zonulin activates protease-activated receptor 2 (PAR2), a member of the G-protein-coupled receptor family and induces signaling events that lead to changes in the actin cytoskeleton and cell-cell junctions [10].

Renal disease, including mesangial IgA deposition without clinically overt IgA nephropathy, has been described in patients with CD [11]. In patients with IgA nephropathy, gluten induces alterations in the intestinal immunity and barrier function $[11,12]$. Withdrawal of gluten from the diet of some individuals with IgA nephropathy and nephrotic syndrome has been associated with resolution of both $\mathrm{CD}$ and proteinuric renal disease [13].

PAR2 is expressed on podocytes, the visceral epithelial cell that contributes to the glomerular filtration barrier. It is plausible that gluten-induced elevation in serum zonulin levels may influence the interaction between PAR2 and PAR3, which has been implicated in cell signaling and the podocyte structure and function [14]. The plasma zonulin concentration in children with nephrotic syndrome due to minimal change disease or focal segmental glomerulosclerosis is elevated compared to the circulating levels in healthy controls [15]. A zonulin knock-in mouse model with elevated serum zonulin levels develops albuminuria (unpublished data). A GFD has been documented to be effective in a retrospective case series of children with difficult-to-manage nephrotic syndrome [16]. Taken together, this suggests that there may be a shared biologic basis for disturbances in epithelial integrity in the gut and kidney. However, despite the described association between $\mathrm{CD}$ and specific glomerular disorders, there has been no systematic investigation of albuminuria in a well-characterized cohort of pediatric patients with $\mathrm{CD}$. Therefore, we conducted the following study to test the hypothesis that children and adolescents with newly diagnosed and untreated CD have mild glomerular dysfunction as demonstrated by low-grade albuminuria and that institution of a GFD leads to reduced urinary excretion of albumin.

\section{Methods}

\section{Patients}

Patients were recruited from the outpatient gastroenterology clinic. They presented with gastrointestinal complaints and underwent an evaluation for possible $\mathrm{CD}$. They were all on a glutencontaining diet. All patients who had abnormal serology testing for $\mathrm{CD}$ underwent upper intestinal endoscopy and biopsies. The diagnosis of $\mathrm{CD}$ was made by standard accepted criteria using the combination of abnormal celiac serological tests, namely, measurement of serum IgA, tissue transglutaminase (tTG), and anti-gliadin peptide IgG and IgA levels and endoscopic biopsy findings of characteristic duodenal involvement [17]. Children who had both abnormal serology and biopsies were considered to have $\mathrm{CD}$. Patients who had normal duodenal biopsies as well as unremarkable celiac serology and who had no evidence of active inflammation were used as controls.

\section{Clinical and Laboratory Procedures}

A first morning urine specimen was collected from all children at the initial evaluation. Patients with CD were then started on a GFD. An additional first morning urine specimen was collected at the time of the follow-up clinical assessment that was routinely conducted to assess the clinical response to the GFD.

CD serology tests were performed in the NYU Clinical Chemistry Laboratory. Measurements of urine albumin and Cr concentrations were performed in the clinical chemistry laboratory. Albuminuria was measured using an ELISA kit and expressed as the albumin: Cr ratio ( $\mu \mathrm{g} / \mathrm{mg} \mathrm{Cr}$ ) (ACR). The estimated glomerular filtration rate was calculated using the modified bedside Schwartz formula [18].

Five milliliters of whole blood was obtained for evaluation of genetic markers and analyzed for Genetic markers HLA-DQ2/ DQ8 haplotypes by Prometheus Laboratories. PROMETHEUS ${ }^{\circledR}$ Celiac Genetics analyzed patients' genetic profile for genes specifically associated with CD. Advanced high-resolution DNA probe analysis of HLA-DQ haplotypes allows for disease risk stratification depending upon which HLA genes are detected. Patients were classified as categories 1-8 based on their DQ genotype, with category 1 being extremely low risk (DQ2- and DQ8-) and category 8 being extremely high risk (DQ2 homozygous).

\section{Statistical Methods}

Clinical and laboratory data were obtained from the electronic health record. The initial values in the patients with CD were compared to controls by the $t$ test. In addition, the effect of a GFD was assessed with a paired $t$ test comparing the initial level of albuminuria in each child with newly diagnosed CD to the follow-up urine determination in the same child. Results are provided as mean \pm 
Table 1. Clinical characteristics of CD patients

\begin{tabular}{lccc}
\hline & $\begin{array}{l}\text { Total cohort } \\
(n=46)\end{array}$ & $\begin{array}{l}\text { Follow-up subgroup } \\
(n=17)\end{array}$ & $p$ value \\
\hline Age, years & $9.4 \pm 0.6$ & $9.1 \pm 1.1$ & 0.79 \\
Gender (M:F) & $22 \mathrm{M}: 24 \mathrm{~F}$ & $8 \mathrm{M}: 9 \mathrm{~F}$ & 1.00 \\
Race & 46 white & 17 white & 1.00 \\
Ethnicity & 2 Hispanic & 0 Hispanic & 1.00 \\
BMI, kg/m & $16.8 \pm 0.8$ & $17.3 \pm 1.7$ & 0.72 \\
BP systolic, mm Hg & $102.9 \pm 1.4$ & $103.3 \pm 2.6$ & 0.90 \\
BP diastolic, mm Hg & $61.1 \pm 1.1$ & $60.7 \pm 1.6$ & 0.84 \\
Serum Cr, mg/dL & $0.48 \pm 0.02$ & $0.45 \pm 0.03$ & 0.48 \\
eGFR, mL/min/1.73 m ${ }^{2}$ & $117 \pm 3$ & $121 \pm 4$ & 0.54 \\
Serum albumin, g/dL & $4.5 \pm 0.1$ & $4.3 \pm 0.1$ & 0.12 \\
tTG $^{\mathrm{a}}$ & $16.5 \pm 2.0$ & $19.0 \pm 3.5$ & 0.73 \\
\hline
\end{tabular}

CD, celiac disease; tTG, tissue transglutaminase; eGFR, estimated glomerular filtration rate. ${ }^{a}$ The tTG serum levels are expressed as the level/upper limit of normal for the laboratory performing test.
SEM. The study was approved by the Institutional Review Board of NYU Langone Health and NYU Grossman School of Medicine (Protocol number: i15-00616 CR6).

\section{Results}

\section{Patients}

One hundred children being evaluated for possible CD were screened for this study. Four children did not supply urine for ACR determination. Six children were excluded from the study because the diagnosis of CD was indeterminate; specifically, 5 had elevated tTG IgA and 1 had IgA deficiency but the duodenal biopsies were not consistent with CD. Twenty-three control patients who had evidence of other gastrointestinal inflammatory disorders were excluded. The remaining 67 children were included in the study.

\section{Initial Evaluation}

Forty-six children (age $9.4 \pm 0.6$ [range: 2.6-17.5] years; 22 M: 24 F; BMI $16.8 \pm 0.8$ ) with newly diagnosed $\mathrm{CD}$ and 21 control children who had no evidence of active inflammation (age $10.1 \pm 1.1$ [range: 2.6-18.6] years; $10 \mathrm{M}: 11 \mathrm{~F}, \mathrm{BMI} 16.0 \pm 1.6)$ had urine analyzed for albuminuria. There was no difference in the age $(p=0.54)$ and BMI $(p=0.66)$ between the 2 groups. The ACR in the children with newly diagnosed CD was $20.2 \pm 5.6$ (7 children had values $\geq 30 \mu \mathrm{g} / \mathrm{mg}$ ) compared with $8.4 \pm 1.1 \mu \mathrm{g} /$ mg ( 0 children $\geq 30 \mu \mathrm{g} / \mathrm{mg}$ ) in the controls, $p=0.16$. The level exceeded the cutoff for microalbuminuria $(30 \mu \mathrm{g} /$ $\mathrm{mg}$ ) in $7 / 46$ of the children with CD compared to $0 / 21$ control cases, $p=0.09$.

The clinical characteristics of the children with $\mathrm{CD}$ who were included in this study are summarized in the Table 1. The subgroup with serial assessment of ACR in response to treatment with a GFD (see below) was comparable to the complete cohort of children with newly diagnosed CD.

\section{Effect of a GFD}

Seventeen patients with CD (8 M:9 F), all white, mean age $9.1 \pm 1.1$ (range: 2.6-17.4) years, had a follow-up assessment to evaluate the clinical efficacy of the GFD. Sixteen patients had elevated tTG IgA and the other patients had IgA deficiency with an elevated tTG IgG. At diagnosis, 1 child was receiving escitalopram and a second recombinant human growth hormone. After the initial assessment, 2 patients took lansoprazole, but it was discontinued 2 months before the follow-up evaluation. None were receiving immunosuppressive medications. $\mathrm{BP}$, serum $\mathrm{Cr}$, and albumin concentrations were normal in all patients. The estimated glomerular filtration rate was normal $\left(>90 \mathrm{~mL} / \mathrm{min} / 1.73 \mathrm{~m}^{2}\right)$ in all but 4 patients, in whom it was $74,83,87$, and $89 \mathrm{~mL} / \mathrm{min} / 1.73 \mathrm{~m}^{2}$. These children had CKD stage 2 and had no other clinical evidence of underlying renal disease. There was no difference in the age, BMI, and serum tTG levels of the 17 children who had follow-up urine determinations compared with the remaining 29 children with $\mathrm{CD}$ from whom we were unable to obtain follow-up urine for ACR (age $p=$ 0.69 ; BMI $p=0.60$; tTG $p=0.65$ ). 


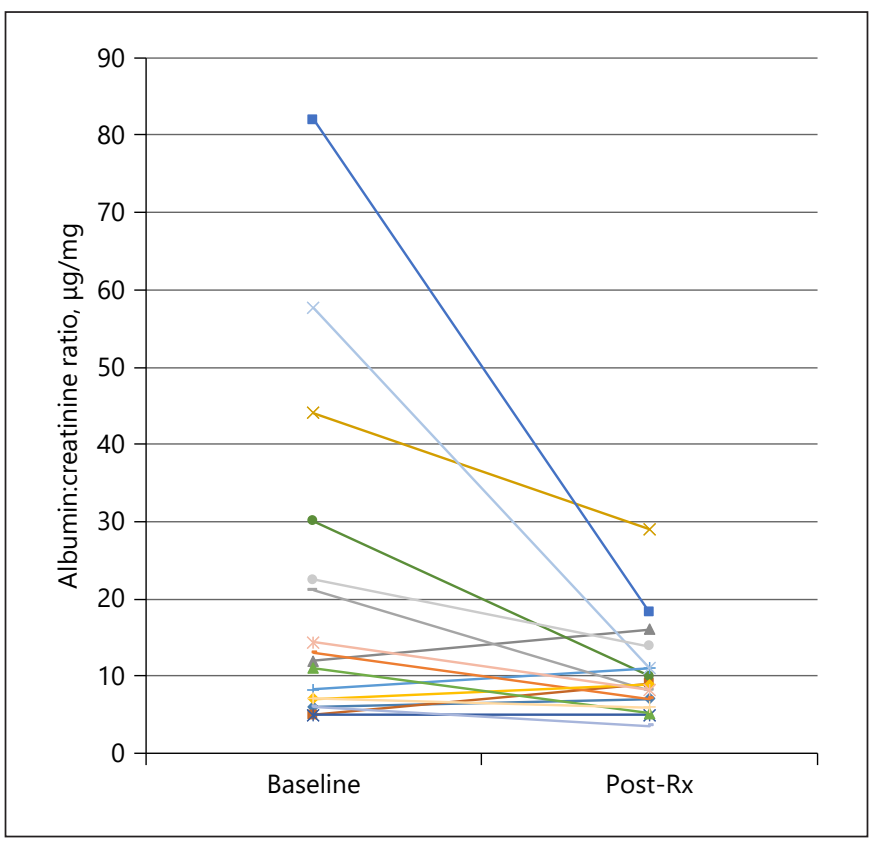

Fig. 1. Paired ACR levels in children with newly diagnosed $C D$ at diagnosis and after treatment with a GFD. ACR, albumin: Cr ratio; $\mathrm{CD}$, celiac disease; GFD, gluten-free diet.

The mean interval on the GFD was $6.1 \pm 0.7$ months. As a measure of compliance with the GFD, initial tTG levels in these 17 children (expressed as the level/upper limit of normal for the laboratory performing test) were compared to the value obtained at the follow-up visit. In this group, the initial tTG was $19.0 \pm 3.5$ compared to 5.0 \pm 1.9 at follow-up, $p=0.0007$. There was a decline in the serum tTG concentration in 16 out of 17 patients. The initial ACR in these 17 children was $20.7 \pm 5.2$ (4 had levels $\geq 30 \mu \mathrm{g} / \mathrm{mg}$ ) that fell to $10.4 \pm 1.5 \mu \mathrm{g} / \mathrm{mg}$ at the repeat evaluation (none of the levels were $\geq 30 \mu \mathrm{g} / \mathrm{mg}$ ), $p=0.035$ (Fig. 1). The percentage decline in albuminuria is illustrated in Figure 2. Using $10 \mu \mathrm{g} / \mathrm{mg}$ as a cutoff value for ACR, 2 out of 7 children whose initial level was below this level had a decline in albuminuria, compared to 9 out of 10 patients whose $A C R \geq 10 \mu \mathrm{g} / \mathrm{mg}, p=0.035$. There was no relationship between adherence to the GFD and the percentage decline in albuminuria. The genetic risk category was assessed in 11 out of the 17 children with paired ACR samples. The category was numerically higher in children with an ACR $\geq 10 \mu \mathrm{g} / \mathrm{mg}, 6.25 \pm 0.86$ versus those patients below that threshold, $4.67 \pm 0.67$, not significant, $p=0.32$.

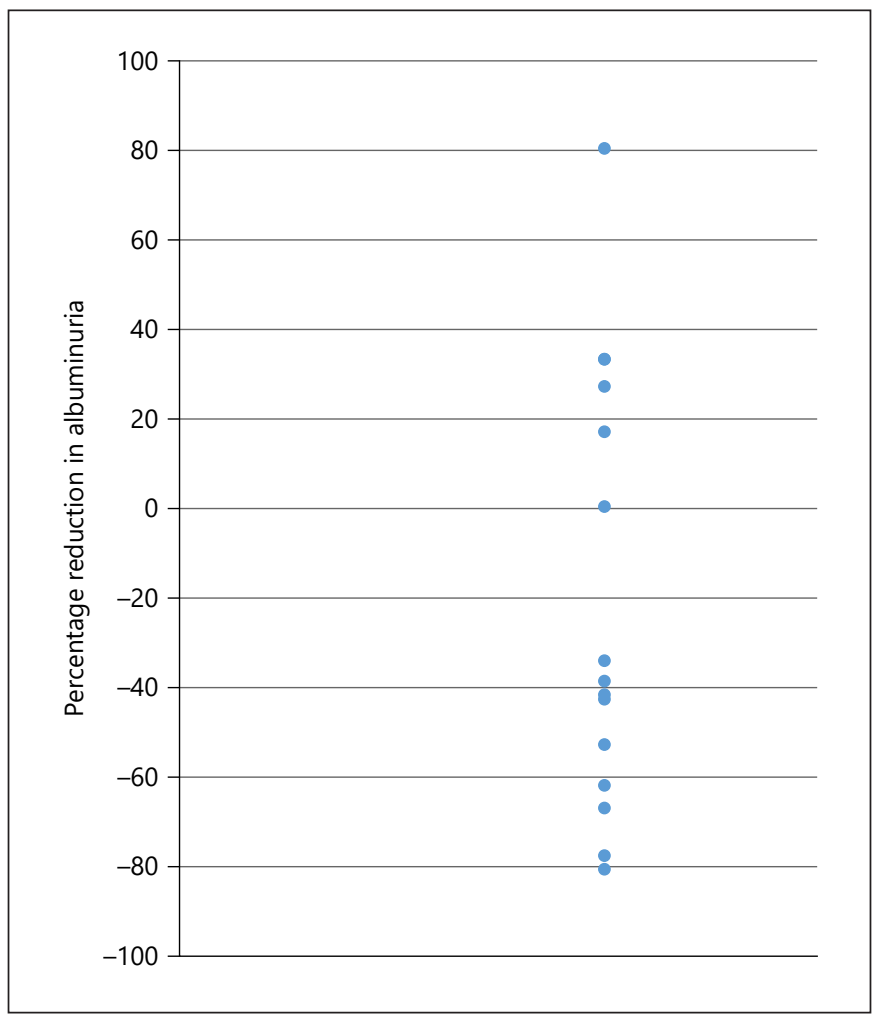

Fig. 2. Percentage reduction in albuminuria based on paired ACR levels in children with newly diagnosed $C D$ at diagnosis and after treatment with a GFD. ACR, albumin: $\mathrm{Cr}$ ratio; $\mathrm{CD}$, celiac disease; GFD, gluten-free diet.

\section{Discussion}

In this prospective, single-center, open label study, we have demonstrated that at diagnosis, a subset of children and adolescents with newly diagnosed CD have albuminuria. In 7 out of 46 patients, the albuminuria exceeded the cutoff value for microalbuminuria established in diabetic nephropathy ( $\mathrm{ACR} \geq 30 \mu \mathrm{g} / \mathrm{mg}$ ), underscoring the clinical relevance of our findings. Regardless of the baseline level, the amount of albuminuria was reduced by $50 \%$ in response to the implementation of a GFD in the overall group placed on a GFD with the change more consistent in the subgroup of children with a baseline ACR $\geq 10 \mu \mathrm{g} /$ $\mathrm{mL}$. Considering the time frame of our observations, it is unlikely that there was any permanent morphological change in the podocyte or cell loss. Instead, we propose that there is reversible change in podocyte integrity and normalization of the glomerular filtration barrier that is achieved rapidly with the implementation of a GFD. However, because we did not collect serial urine samples 
during the initial phase of therapy, we cannot comment on the kinetics of the response to the change in diet.

$\mathrm{CD}$ has been linked to glomerular disease in the past. There are isolated cases, in which CD has occurred in association with dermatological findings and a renal biopsy demonstrated changes consistent with membranous nephropathy or membranoproliferative glomerulonephritis [19-21]. The most common kidney disease association is with IgA nephropathy $[11,12]$. Under these circumstances, the pathogenesis is presumed to represent a primary defect in the gut epithelium. Increased gastrointestinal permeability leads to sensitization to food items, local production of IgA that enters the circulation, the formation of circulating immune complexes, and deposition of the complexes in the mesangial regions of the glomerulus. The deposition of IgG-IgA complexes leads to inflammation with hematuria and proteinuria.

A more direct link between CD and albuminuria may be the increased levels of zonulin that alter gastrointestinal permeability and that may bind to the PAR2 receptor on the podocyte cell membrane to increase the glomerular permeability. Further studies are needed to assess the relationship between plasma zonulin levels and albuminuria in patients with CD. It should be acknowledged that the zonulin level in the systemic circulation may not reflect the concentration in the glomerular microcirculation due to clearance in the liver, the microalbuminuria in our group of patients with CD exceeded the diabetic cutoff for microalbuminuria, a marker of incipient glomerular dysfunction, in $15 \%$ of the cases. Although the degree of albuminuria observed in our patients had no short-term effect on the kidney function, there may be long-term ramifications related to sustained albuminuria to the degree that we documented. In adults, even low levels of albuminuria are associated with increased risk of developing major adverse cardiovascular events [22]. Thus, there is no truly safe level of albuminuria. This raises the question that inadequate control of $\mathrm{CD}$ because of suboptimal adherence to a GFD may increase albuminuria and the risk of a major adverse cardiovascular event during adulthood [22]. There are studies suggestive of increased risk of CV disease in patients with CD [23]. Future studies in adults with CD who are followed up for more extended periods of time will be needed to determine if this risk is linked to albuminuria.

There are potential clinical implications of our findings. Generally, the response to a GFD is assessed based on clinical symptoms and resolution of serologic abnormalities. If changes in albuminuria are an immediate reversible response to a GFD, they may be a useful short-

Albuminuria and Gluten-Free Diet in Celiac Disease term indicator of adherence to the diet and resolution of the disease complex. Incorporation of routine assessment of albuminuria into the clinical monitoring of children with CD may provide an early biomarker of disease relapse and/or incomplete adherence to the dietary intervention. With regard to measurement of serum zonulin levels, it has been demonstrated that the circulating concentration of zonulin declines in response to the implementation of a GFD in patients with CD [9, 24]. Further work is needed to determine if changes in albuminuria are associated with the magnitude of reduction in serum zonulin concentration. In addition, clinical investigation of other gastrointestinal disorders is required to determine whether they are also associated with zonulin release and subsequent glomerular dysfunction and albuminuria.

The limitations of this study include the relatively small sample size, the lack of serial urine specimens during the initial dietary treatment, the inability to correlate the albuminuria with the gastrointestinal morphology, and the lack of renal tissue to assess, whether there were persistent glomerular structural changes. In the absence of detailed data about dietary intake, we cannot exclude the possible impact of altered protein consumption as a consequence of the GFD on urinary albumin excretion. In addition, we cannot confirm the specificity of the beneficial effect of the GFD on albuminuria in the children with $\mathrm{CD}$. Future studies that assess the temporal course of albuminuria following implementation of a GFD and evaluation of albuminuria in response to other treatments of gastrointestinal disorders in children will help answer this question. The level of albuminuria in our patients, those with CD and the controls, was comparable to other studies that measured albuminuria in children [25]. However, the level of albuminuria at diagnosis is less relevant than the decline that we noted in patients, whose baseline ACR was $\geq 10 \mu \mathrm{g} / \mathrm{mg}$ after implementation of the GFD. We are unable to explain why some children with newly diagnosed CD had microalbuminuria, while others did not. Our preliminary data suggest that the genetic risk score may be higher in CD patients with albuminuria $>10$ $\mu \mathrm{g} / \mathrm{mL}$. We have recently demonstrated that the degree of duodenal involvement in children with CD significantly correlated with the genetic risk score [26]. It is interesting to speculate that the increased duodenal involvement is associated with an increased risk of albuminuria. However, this will require confirmation in a larger cohort of patients. We are unable to address whether the changes in albuminuria are persistent and whether they fluctuate in response to adherence to the GFD. Finally, measuring 
plasma zonulin levels in these children may help explain the association of albuminuria with $\mathrm{CD}$ and may also allow for risk stratification for albuminuria in children with newly diagnosed CD.

In summary, pediatric patients with newly diagnosed CD have low-grade albuminuria that is numerically higher, but not statistically significant, compared to healthy controls. The level exceeds the cutoff value for microalbuminuria in patients with diabetes in $15 \%$ of the patients. There was a consistent $50 \%$ reduction in albuminuria after implementation of a GFD in the overall group that was more pronounced in the subgroup with a baseline ACR $\geq 10 \mu \mathrm{g} / \mathrm{mg}$. These findings suggest that CD can have adverse effects on the glomerular barrier function that are reversible with a GFD. Collaborative efforts are required to clarify whether routine monitoring of albuminuria should be incorporated into the clinical care of patients with $\mathrm{CD}$ and to assess the long-term health ramifications of the albuminuria on cardiovascular outcomes. Future studies are warranted to assess the impact of a GFD on glomerular diseases such as IgA nephropathy that are linked to the gastrointestinal tract and others like focal segmental glomerulosclerosis and membranous nephropathy that are associated with higher levels of proteinuria.

\section{Acknowledgements}

The authors thank the patients for participating in this study.

\section{Statement of Ethics}

This study is in compliance with the guidelines for human studies and was conducted ethically in accordance with the World Medical Association Declaration of Helsinki. The study was approved by the NYU Grossman School of Medicine Institutional Review Board (Protocol number: i15-00616 CR6). Parents or guardians gave their written informed consent and participants provided assent.

\section{Conflict of Interest Statement}

The authors have no conflicts of interest to disclose.

\section{Funding Sources}

This work was supported in part by Grant DK100307 (HT)

\section{Author Contributions}

Jeremiah Levine: study concept and design, recruited patients, acquisition of data, study supervision, analysis and interpretation of data, drafting of the manuscript, and statistical analysis. Leora Hauptman: acquisition of data and monitored adherence to the diet. Libia Moy: recruited patients, acquisition of data, and study supervision. Howard Trachtman: study concept and design, analysis and interpretation of data, drafting of the manuscript, and statistical analysis. All authors reviewed and approved the final version of the manuscript as submitted and agreed to be accountable for all aspects of the work.

\section{References}

1 Ludvigsson JF, Leffler DA, Bai JC, Biagi JC, Fasano A, Green PH, et al. The Oslo definitions for coeliac disease and related terms. Gut. 2013;62:43-52.

2 Singh P, Arora A, Strand TA, Leffler DA, Catassi C, Green PH, et al. Global prevalence of celiac disease: systemic review and meta-analysis. Clinical Gastroenterol Hepatol. 2018;16: 823-36.

3 De Silvestri A, Capittini C, Poddighe D, Valsecchi C, Marseglia G, Tagliacarne SC, et al. HLA-DQ genetics in children with celiac disease: a meta-analysis suggesting a two-step genetic screening procedure starting with HLA-DQ $\beta$ chains. Pediatr Res. 2018;83:56472.

4 Pietzak MM, Schofield TC, McGinniss MJ, Nakamura RM. Stratifying risk for celiac disease in a large at-risk United States population by using HLA alleles. Clin Gastroenterol Heptol. 2009;7:966-71.

5 Meresse B, Malamut G, Cerf-Bensussan N. Celiac disease: an immunological jigsaw. Immunity. 2012;36(6):907-19.
6 Lammers KM, Lu R, Brownley J, Lu B, Gerard $\mathrm{C}$, Thomas $\mathrm{K}$, et al. Gliadin induces an increase in intestinal permeability and zonulin release by binding to the chemokine receptor CXCR3. Gastroenterology. 2008;135(1):194204.e3.

7 Bischoff SC, Barbara G, Buurman W, Ockhuizen T, Schulzke JD, Serino M, et al. Intestinal permeability:-a new target for disease prevention and therapy. BMC Gastroenterol. 2014; 14:189.

8 van Leeuwen MA, Lindenbergh-Kortleve DJ, Raatgeep HC, de Ruiter LF, de Krijger RR, Groeneweg $\mathrm{M}$, et al. Increased production of interleukin-21, but not interleukin-17A, in the small intestine characterizes pediatric celiac disease. Mucosal Immunol. 2013;6(6): 1202-13.

9 Fasano A. Zonulin and its regulation of intestinal barrier function: the biological door to inflammation, autoimmunity, and cancer. Physiol Rev. 2011;91(1):151-75.
10 Tripathi A, Lammers KM, Goldblum S, SheaDonohue T, Netzel-Arnett S, Buzza MS, et al. Identification of human zonulin, a physiological modulator of tight junctions, as prehaptoglobin-2. Proc Natl Acad Sci U S A. 2009; 106(39):16799-804.

11 Coppo R. The intestine-renal connection in IgA nephropathy. Nephrol Dial Transplant. 2015;30(3):360-6.

12 Rostoker G, Chaumette MT, Wirquin E, Delchier JC, Petit-Phar M, Andre C, et al. IgA mesangial nephritis, IgA antigliadin antibodies, and coeliac disease. Lancet. 1990; 336(8718):824-5.

13 Woodrow G, Innes A, Boyd SM, Burden RP. A case of IgA nephropathy with coeliac disease responding to a gluten-free diet. Nephrol Dial Transplant. 1993;8(12):1382-3.

14 Madhusudhan T, Wang H, Straub BK, Gröne E, Zhou Q, Shahzad K, et al. Cytoprotective signaling by activated protein $\mathrm{C}$ requires protease-activated receptor-3 in podocytes. Blood. 2012;119(3):874-83. 
15 Trachtman H, Gipson DS, Lemley KV, Troost JP, Faul C, Morrison DJ, et al. Plasma zonulin levels in childhood nephrotic syndrome. Front Pediatr. 2019;7:197.

16 Lemley KV, Faul C, Schramm K, Meyers K, Kaskel F, Dell KM, et al. The effect of a glutenfree diet in children with difficult-to-manage nephrotic syndrome. Pediatrics. 2016 Jul; 138(1):e20154528..).

17 Dickson BC, Streutker CJ, Chetty R. Coeliac disease: an update for pathologists. J Clin Pathol. 2006;59(10):1008-16.

18 Schwartz GJ, Muñoz A, Schneider MF, Mak RH, Kaskel F, Warady BA, et al. New equations to estimate GFR in children with CKD. J Am Soc Nephrol. 2009;20(3):629-37.

19 Politis EE, Droulias J, Nikolopoulou N, Hadjiconstantinou V, Apostolou T. Coexistent membranous nephropathy and celiac disease in a 40-year old male. Clin Nephrol. 2009; 71(1):99-100.
20 Sacks SH, Bushell A, Rust NA, Karagiannis JA, Jewell DP, Ledingham JG, et al. Functional and biochemical subtypes of the haplotype HLA-DR3 in patients with celiac disease or idiopathic membranous nephropathy. Hum Immunol. 1987;20(2):175-87.

21 Jhaveri KD, D'Agati VD, Pursell R, Serur D. Coeliac sprue-associated membranoproliferative glomerulonephritis (MPGN). Nephrol Dial Transplant. 2009;24(11):3545-8.

22 Ruggenenti P, Porrini E, Motterlini N, Perna A, Ilieva AP, Iliev IP, et al. Measurable urinary albumin predicts cardiovascular risk among normoalbuminuric patients with type 2 diabetes. J Am Soc Nephrol. 2012;23(10):171724.
23 Gajulapalli RD, Pattanshetty DJ. Risk of coronary artery disease in celiac disease population. Saudi J Gastroenterol. 2017;23(4):253-8.

24 Fasano A, Not T, Wang W, Uzzau S, Berti I, Tommasini A, et al. Zonulin, a newly discovered modulator of intestinal permeability, and its expression in coeliac disease. Lancet. 2000;355(9214):1518-9.

25 Lehrnbecher T, Greissinger S, Navid F, Pfüller $\mathrm{H}$, Jeschke R. Albumin, IgG, retinol-binding protein, and alpha1-microglobulin excretion in childhood. Pediatr Nephrol. 1998;12(4): 290-2.

26 Levine JJ, Gaylord A, Thomas KM, Moy L, Trasande L. Tu1330: factors associated with degree of duodenal inflammation in childen with newly-diagnosed celiac disease. Gastroenterology. 2019;156(6):S1028-9. 